\title{
ON DIMENSIONS OF BLOCK ALGEBRAS
}

\author{
MARKUS LINCKELMANN
}

\begin{abstract}
Following a question by B. Külshammer, we show that an inequality, due to Brauer, involving the dimension of a block algebra, has an analogue for source algebras, and use this to show that a certain case where this inequality is an equality can be characterised in terms of the structure of the source algebra, generalising a similar result on blocks of minimal dimensions.
\end{abstract}

Let $p$ be a prime and $k$ an algebraically closed field of characteristic $p$. Let $G$ be a finite group and $B$ a block algebra of $k G$; that is, $B$ is an indecomposable direct factor of $k G$ as $k$-algebra. By a result of Brauer in [2], the dimension of $B$ satisfies the inequality

$$
\operatorname{dim}_{k}(B) \geq p^{2 a-d} \cdot \ell(B) \cdot u_{B}^{2}
$$

where $p^{a}$ is the order of a Sylow-p-subgroup of $G, p^{d}$ is the order of a defect group of $B$, $\ell(B)$ is the number of isomorphism classes of simple $B$-modules and $u_{B}$ is the unique positive integer such that $p^{a-d} \cdot u_{B}$ is the greatest common divisor of the dimensions of the simple $B$-modules. It is well-known that $u_{B}$ is prime to $p$. Külshammer raised the question whether an equality could be expressed in terms of the structure of a source algebra of $B$, generalising the result in [3] on blocks of minimal dimension. We show that this is the case. The first observation is an analogue for source algebras of Brauer's inequality. We keep the notation above and refer to [5] for block theoretic background material.

Theorem 1. Let $A$ be a source algebra of $B$. Then $\operatorname{dim}_{k}(A) \geq p^{d} \cdot \ell(B) \cdot u_{A}^{2}$, where $u_{A}$ is the greatest common divisor of the dimensions of the simple A-modules.

Proof. One can prove this by adapting Brauer's proof of [2, Theorem 1]. Alternatively, one can use the theorem of Wedderburn-Malcev, according to which $A$ contains a unitary subalgebra isomorphic to $A / J(A)$, where $J(A)$ denotes the Jacobson radical of $A$. Every indecomposable factor of $A / J(A)$ is a matrix algebra of dimension $n^{2}$ for some integer $n$ which is divisible by $u_{A}$, hence of the form $T \otimes_{k} S$ for some matrix algebra $T$ of dimension $u_{A}^{2}$ and some matrix algebra $S$ of dimension $\frac{n^{2}}{u_{A}^{2}}$. Thus $A$ contains $T$ as unitary subalgebra, hence $A \cong T \otimes_{k} A^{\prime}$, where $A^{\prime}$ is the centraliser of $T$ in $A$. Note that $B, A, A^{\prime}$ are Morita equivalent; in particular $\ell(B)=\ell(A)=\ell\left(A^{\prime}\right)$. Since $A$ is projective as module over a defect group, every projective indecomposable $A$ module has dimension divisible by $p^{d}$. By [5, Corollary (44.9)], the integer $u_{A}$ is prime to $p$. Thus every projective $A^{\prime}$-module has still dimension divisible by $p^{d}$. Since $A^{\prime}$ is a direct sum, as left $A^{\prime}$-module, of at least $\ell\left(A^{\prime}\right)$ indecomposable direct summands, it follows that $\operatorname{dim}_{k}\left(A^{\prime}\right) \geq p^{d} \cdot \ell(B)$, hence $\operatorname{dim}_{k}(A)=\operatorname{dim}_{k}\left(A^{\prime}\right) \cdot u_{A}^{2} \geq p^{d} \cdot \ell(B) \cdot u_{A}^{2}$ as claimed.

Received by the editors June 2, 2009. 
An equality $\operatorname{dim}_{k}(A)=p^{d} \cdot \ell(B) \cdot u_{A}^{2}$ does not seem to imply strong structural conditions on $A$; for instance, if $\ell(B)=1$ then $\operatorname{dim}_{k}(A)=p^{d} \cdot u_{A}^{2}$. Indeed, if $\ell(B)=1$ then $u_{A}$ is the dimension of the unique (up to isomorphism) simple $A$-module, and hence the algebra $A^{\prime}$ arising in the proof of Theorem 1 is a basic algebra, hence local, and thus its dimension is the unique Cartan invariant $p^{d}$ of $B$. If one requires $u_{A}=1$, one gets the following structural characterisation:

Theorem 2. Let $A$ be a source algebra of $B$ with defect group P. The following are equivalent.

(i) $\operatorname{dim}_{k}(A)=p^{d} \cdot \ell(B)$.

(ii) $A \cong k(P \rtimes E)$ for some abelian $p^{\prime}$-subgroup $E$ of $\operatorname{Aut}(P)$.

Proof. Suppose that (i) holds. Let $J$ be a primitive decomposition of $1_{A}$ in $A$; that is, $J$ is a set of primitive pairwise orthogonal idempotents in $A$ such that $1_{A}=\sum_{j \in J} j$. Then $A=\oplus_{j \in J} A j$ as left $A$-module, and $A j$ is projective as $k P$-module for each $j \in J$. Thus $\operatorname{dim}_{k}(A)=p^{d} \sum_{j \in J} \frac{\operatorname{dim}_{k}(A j)}{p^{d}}$. The equality in (i) is therefore equivalent to $|J|=\ell(B)$ and $\operatorname{dim}_{k}(A j)=p^{d}$ for all $j \in J$. Thus each point (cf. [5, §4]) of $A$ has multiplicity 1 , or equivalently, $A$ is a basic $k$-algebra, hence each simple $A$ module has dimension 1, and each $A j$ restricted to $k P$ is isomorphic to the regular module $k P$. Thus the radical of $A j$ as $A$-module is equal to the radical of $A j$ as $k P$-module, hence $J(k P) A=J(A)$. The same argument yields $J(A)=A J(k P)$. By a result of Puig (cf. $[4,14.6]$ or $[5$, Theorem (44.3)] or also [1] for another proof), as $k P$ - $k P$-bimodule, $A$ is isomorphic to a direct sum of $k(P \rtimes E)$ for some $p^{\prime}$-subgroup $E$ of $\operatorname{Aut}(P)$ and indecomposable direct summands of the form $k P \otimes_{k Q} \varphi k P$, where $Q$ is a proper subgroup of $P$ and $\varphi: Q \rightarrow P$ is an injective group homomorphism. The equality $J(k P) A=A J(k P)$ forces that there is no summand of that form. To see this, note first that $J(k P) A$ is a $k P-k P$-submodule of $A$, thus so is the quotient $A / J(k P) A$. The elements of $P$ act as identity on the left of $A / J(k P) A$, hence also on the right, but the elements in $P$ outside of $\varphi(Q)$ do not act as identity on the right side of the $k P$ - $k P$-bimodule $\left.\left(k P \otimes_{k Q} \varphi P\right) / J(k P)\left(k P \otimes_{k Q}{ } k P\right)\right) \cong k \otimes_{k Q} \varphi k P$. This implies that $A=k(P \rtimes E)$ as $k P$ - $k P$-bimodule. But then, by [4, 14.6] again (or the alternative references [1, Theorem 1], [5, Theorem (45.11)]), $A$ is isomorphic, as interior $P$-algebra (cf. $[5, \S 10])$, to a twisted group algebra $k_{\alpha}(P \rtimes E)$, for some $\alpha \in H^{2}\left(E ; k^{\times}\right)$, inflated trivially to $P \rtimes E$. The fact that $A$ has a simple module of dimension 1 implies that there is an algebra homomorphism $\epsilon: k_{\alpha} E \rightarrow k$. If $x, y \in E$ their product in $k_{\alpha} E$ is $x \cdot y=\alpha(x, y) x y$, where $x y$ is the product of $x, y$ in $E$. Thus $x \cdot y$ is mapped under $\epsilon$ to $\epsilon(x) \epsilon(y)=\alpha(x, y) \epsilon(x y)$, proving that $\alpha$ is a 2-coboundary, or equivalently, that its class is zero. But then, since every simple $A$-module has dimension 1 this also forces $E$ to be abelian. Thus (i) implies (ii). The converse is easy.

A block $B$ satisfying the equivalent conditions of Theorem 2 is splendidly Morita equivalent to its Brauer correspondent, hence satisfies in particular all relevant conjectures by Alperin, Broué (for $P$ abelian), Dade and Robinson. For $S$ a subgroup of $G$ we denote as usual by $(k G)^{S}$ the subalgebra of $k G$ consisting of all elements in $k G$ which are invariant under conjugation by elements in $S$. 
Theorem 3. Let $A$ be a source algebra of $B$. We have $\operatorname{dim}_{k}(B) \geq p^{2 a-2 d} \operatorname{dim}_{k}(A)$, with equality if and only if the block idempotent $1_{B}$ remains primitive in $(k G)^{S}$ for any Sylow-p-subgroup $S$ of $G$.

Proof. Choose a Sylow-p-subgroup $S$ of $G$ and a defect group $P$ of $B$ such that $P \leq S$. Denote by $\operatorname{Br}_{P}:(k G)^{P} \rightarrow k C_{G}(P)$ the Brauer homomorphism (cf. [5, $\left.\S 11\right]$ ). Since $\operatorname{Br}_{P}\left(1_{B}\right) \neq 0$ there is a primitive idempotent $e \in B^{S}$ such that $\operatorname{Br}_{P}(e) \neq 0$. Thus there is a primitive idempotent $i \in(e B e)^{P}$ such that $\operatorname{Br}_{P}(i) \neq 0$. Then $e$ belongs to a point $\sigma$ of $S$ on $B$ and $i$ belongs to a local point $\gamma$ of $P$ on $B$ such that $P_{\gamma} \leq S_{\sigma}$ (cf. $[5, \S 13,14]$ for background material on pointed groups, inclusion between pointed groups, and local pointed groups). Since $P$ is maximal with the property $\operatorname{Br}_{P}\left(1_{B}\right) \neq 0$ it follows that $P_{\gamma}$ is a defect pointed group of $S_{\sigma}$, hence $\sigma \subseteq \operatorname{Tr}_{P}^{S}\left(A^{P} \gamma A^{P}\right)$ (cf. [5, Proposition (18.5)]). Thus, by Higman's criterion (cf. [5, Corollary (17.3)]), as $k(G \times S)$-module, $k G e$ is relatively $G \times P$-projective, and Green's indecomposablity theorem [5, Corollary (23.5)] implies therefore that $k G e \cong k G i \otimes_{k P}$ $k S$ as $k(G \times S)$-modules. The $k$-dual of $k G i \otimes_{k P} k S$ is isomorphic to the $k(S \times G)$ module $k S \otimes_{k P} i k G$. Tensoring these two modules over $k G$ yields an isomorphism of $k S$ - $k S$-bimodules $e B e \cong k S \otimes_{k P} i k G i \otimes_{k P} k S$. Since $\operatorname{Br}_{P}(i) \neq 0$, the algebra $i k G i=i B i$ is a source algebra of $B$; in particular, $\operatorname{dim}_{k}(i k G i)=\operatorname{dim}_{k}(A)$. Clearly $\operatorname{dim}_{k}\left(k S \otimes_{k P} i k G i \otimes_{k P} k S\right)=p^{2 a-2 d} \operatorname{dim}_{k}(A)$ and $\operatorname{dim}_{k}(B) \geq \operatorname{dim}_{k}(e B e)$. This shows the inequality, and also shows that the equality holds if and only if $1_{B}=e$ is primitive in $(k G)^{S}$. Since $1_{B}$ is a central idempotent, $1_{B}$ is then primitive in $(k G)^{S^{\prime}}$ for any Sylow-p-subgroup $S^{\prime}$ of $G$, whence the second statement.

The result in [3] on blocks of minimal dimension follows easily from the above:

Corollary 4 ([3, Theorem $])$. If $\operatorname{dim}_{k}(B)=p^{2 a-d}$ then $B$ is a nilpotent block with source algebra $k P$, where $P$ is a defect group of $B$.

Proof. Combining the above Theorem 3 and Theorem 1 yields in particular

$$
\operatorname{dim}_{k}(B) \geq p^{2 a-2 d} \operatorname{dim}_{k}(A) \geq p^{2 a-d} \ell(B)
$$

Thus the equality $\operatorname{dim}_{k}(B)=p^{2 a-d}$ forces $\ell(B)=1$ and $\operatorname{dim}_{k}(A)=p^{d}$. Since by Theorem 2 also $A \cong k(P \rtimes E)$ for some abelian $p^{\prime}$-group $E$, this implies $E=1$, hence $k P$ is a source algebra of $B$, and in particular, $B$ is nilpotent (cf. [5, Remark $(50.10)])$.

As pointed out by the referee, combining Theorem 1 and Theorem 3 yields the inequality $\operatorname{dim}_{k}(B) \geq p^{2 a-d} \cdot \ell(B) \cdot u_{A}^{2}$; that is, Brauer's inequality with $u_{B}$ replaced by $u_{A}$. Since $u_{A}$ can be smaller than $u_{B}$ this does not imply Brauer's original result. This is due to the fact that the inequality in Theorem 3 does not take into account the multiplicity of a local point of $P$ on $B$. For this reason there is no obvious connection between $u_{A}$ and $u_{B}$. Examples where $u_{A}=1$ and $u_{B}$ is an arbitrary $p^{\prime}$-integer arise from blocks of $H \times P$, where $H$ is a finite $p^{\prime}$-group having an ordinary irreducible character of degree $u_{B}$; in such a situation, $u_{B}$ is precisely the multiplicity of the unique point of $P$ on the block algebra under consideration. 


\section{Acknowledgements}

The author would like to thank the referee for many valuable comments.

\section{References}

[1] J. L. Alperin, M. Linckelmann, R. Rouquier, Source algebras and source modules, J. Algebra 239 (2001), 262-271.

[2] R. Brauer, Notes on representations of finite groups, I, J. London Math. Soc. (2), 13 (1976), $162-166$.

[3] M. Linckelmann, Blocks of minimal dimension, Archiv Math. 89 (2007), 311-314.

[4] L. Puig, Pointed groups and construction of modules, J. Algebra 116 (1989), 7-129.

[5] J. Thévenaz, G-Algebras and Modular Representation Theory, Oxford Science Publications, Clarendon Press, Oxford (1995).

Department of Mathematical Sciences,, University of Aberdeen, Meston Building, AbERdeEn, AB24 3UE, United Kingdom

E-mail address: m.linckelmann@abdn.ac.uk 